\title{
Interleukin-6 is associated with acute concussion in military combat personnel
}

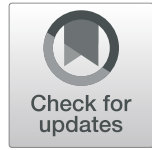

Katie A. Edwards ${ }^{1,2^{*}}$ (D) Jessica M. Gill ${ }^{1,3}$, Cassandra L. Pattinson ${ }^{1}$, Chen Lai ${ }^{1}$, Misha Brière ${ }^{4}$, Nicholas J. Rogers ${ }^{5}$, Denise Milhorn ${ }^{5}$, Jonathan Elliot ${ }^{6}$ and Walter Carr ${ }^{7,8}$

\begin{abstract}
Background: Concussion is the most common type of $\mathrm{TBl}$, yet reliable objective measures related to these injuries and associated recovery processes remain elusive, especially in military personnel. The purpose of this study was to characterize the relationship between cytokines and recovery from acute brain injury in active duty service members. Inflammatory cytokines (IL-6, IL-10, and TNFa) were measured acutely in blood samples within $8 \mathrm{~h}$ following a medically diagnosed concussion and then $24 \mathrm{~h}$ later.

Methods: Participants ( $n=94)$ were categorized into two groups: 1$)$ military personnel who sustained providerdiagnosed concussion, without other major medical diagnosis $(n=45)$ and 2) healthy control participants in the same deployment environment who did not sustain concussion or other illness or injuries $(n=49)$. IL-6, IL-10, and TNFa concentrations were measured using an ultrasensitive single-molecule enzyme-linked immunosorbent assay. Differences in cytokine levels between concussed and healthy groups were evaluated at two time points (time point $1 \leq 8 \mathrm{~h}$ after injury; time point $2=24 \mathrm{~h}$ following time point 1 ).
\end{abstract}

Results: At time point 1, IL-6 median (IQR) concentrations were 2.62 (3.62) in the concussed group, which was greater compared to IL-6 in the healthy control group (1.03 (0.90); $U=420.00, z=-5.12, p<0.001)$. Compared to healthy controls, the concussed group did not differ at time point 1 in IL-10 or TNFa concentrations ( $p$ 's $>0.05$ ). At time point 2, no differences were detected between concussed and healthy controls for IL-6, IL-10, or TNFa ( $p$ 's > 0.05). The median difference between time points 1 and 2 were compared between the concussed and healthy control groups for IL-6, IL-10, and TNFa. Change in IL-6 across time was greater for the concussed group than healthy control $(-1.54$ (3.12); $U=315.00, z=-5.96, p<0.001)$, with no differences between groups in the change of IL-10 or TNFa ( $p$ 's $>0.05)$.

Conclusion: Reported here is a significant elevation of IL-6 levels in concussed military personnel less than $8 \mathrm{~h}$ following injury. Future studies may examine acute and chronic neurological symptomology associated with inflammatory cytokine levels, distinguish individuals at high risk for developing neurological complications, and identify underlying biological pathways to mitigate inflammation and improve outcomes.

Keywords: Inflammatory cytokines, Concussion, Biomarkers

\footnotetext{
*Correspondence: katie.edwards@nih.gov

${ }^{1}$ National Institute of Nursing Research, National Institutes of Health, 3 Center Drive, Building 3, Room 26E, Bethesda, MD 20892, USA

${ }^{2}$ Henry M. Jackson Foundation for the Advancement of Military Medicine, 6720A Rockledge Dr, Bethesda, MD 20817, USA

Full list of author information is available at the end of the article
}

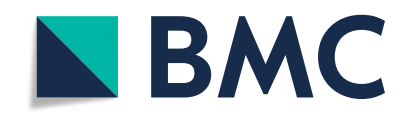

(c) The Author(s). 2020 Open Access This article is licensed under a Creative Commons Attribution 4.0 International License which permits use, sharing, adaptation, distribution and reproduction in any medium or format, as long as you give appropriate credit to the original author(s) and the source, provide a link to the Creative Commons licence, and indicate if changes were made. The images or other third party material in this article are included in the article's Creative Commons licence, unless indicated otherwise in a credit line to the material. If material is not included in the article's Creative Commons licence and your intended use is not permitted by statutory regulation or exceeds the permitted use, you will need to obtain permission directly from the copyright holder. To view a copy of this licence, visit http://creativecommons.org/licenses/by/4.0/. The Creative Commons Public Domain Dedication waiver (http://creativecommons.org/publicdomain/zero/1.0/) applies to the data made available in this article, unless otherwise stated in a credit line to the data. 


\section{Background}

Concussion, or mild traumatic brain injury (mTBI), is the most common type of TBI across all age ranges, yet reliable biomarkers related to these injuries and associated recovery processes remain elusive, especially in military personnel $[1,2]$. At this time, limited objective measures exist for identification of individuals who may be at high risk for developing complications and poor outcomes following concussion. Improving our understanding of underlying biological changes that occur following a concussion or mTBI is crucial to identify people who may be at the most risk of poor outcomes, who require increased monitoring and preventive interventions, and for ongoing monitoring of these individuals [3].

For the military, identifying blood-based markers for concussion has been a 20-year program of research. Concussion is recognized as one of the most prevalent injuries among military members who served in Operation Iraqi Freedom [4] and Operation Enduring Freedom (OEF). A key factor in the rate of concussion among military casualties is adversaries' increasing use of improvised explosive devices (IEDs) [5]. Blast exposure affects multiple organs and tissues, including the central nervous system, which is well documented in preclinical models [6]. Over time, blast exposures as well as blunt force injuries are associated with neurological symptoms that are garnering concern for the health and well-being of military personnel and veterans [7-9].

Blood-based biomarkers show promise in distinguishing patients with traumatic brain injuries who require additional monitoring and interventions, as evidenced by the recent FDA-approval for blood biomarkers ubiquitin C-terminal hydrolase (UCH-L1) and glial fibrillary acidic protein (GFAP) to inform clinical CT decisions [10]. Additional investigation of blood biomarkers related to secondary pathological processes initiated by TBIs may elucidate pathological pathways for research and eventual clinical applications $[11,12]$. Specifically, studies of blood-based inflammatory protein biomarkers implicates underlying inflammatory processes following TBIs of all severities that are important for acute recovery $[13,14]$ and may relate to chronic symptoms $[15,16]$. Not only may inflammatory biomarkers help monitor and predict outcomes, but they also identify inflammatory pathways that could be targeted for therapies [17]. Of key interest are pro- and anti-inflammatory cytokines, as they have been implicated in the underlying balance of inflammatory processes which occur following a TBI $[11,18]$. For example, preclinical brain injury studies of interleukin (IL)-6 indicate some IL-6 activity is beneficial for recruiting immune cells and improving outcomes, especially in the acute phase [19]. However, harmful outcomes may result from either IL-6 deficiency, as demonstrated in
IL-6 knockout mice [20], or chronic IL-6 overexpression $[17,19]$. Likewise, study of IL-10 in preclinical brain injury models has shown poor outcomes in IL-10 knockout mice, with IL-10 administration improving neurological function and decreasing lesion volume [21]. In human studies, inflammatory markers, including levels of IL-6, IL-10, and TNF $\alpha$, are observed to be elevated and associate with poor outcomes in moderate and severe cases of TBI [13, 14, 16, 22-29].

Evaluation of cytokine profiles following concussions are increasing in recent years [30-33]. Previously reported are elevated levels of plasma inflammatory cytokines, IL-6 and TNFa, concurrent with chronic neurological symptomology among military personnel who experienced blunt force and/or blast injury (within 16 months of deployment) [31]. This lab also reported an association between blast exposure and acute increases in levels of IL- 6 and TNF $\alpha$ in an undiagnosed military blast training population [34]. These studies suggest that mild injuries have similar inflammatory changes to preclinical and severe human TBI studies. Concussion is the most common type of TBI and a recognized threat for military personnel deployed to a combat zone; yet, blood levels of IL-6, IL-10, and TNF $\alpha$ have not been studied during the first $24 \mathrm{~h}$ following concussion sustained during a military combat deployment. The previous literature implicates that appropriate inflammatory response is important to successful outcomes; and over or under expression of the cytokine response may contribute to poor outcomes. Thus, protein blood biomarkers, including cytokines, may be useful in monitoring for poor outcomes, which may be especially beneficial among concussed individuals who may not otherwise follow up on mild subjective symptoms [35].

To better understand the role of inflammatory cytokines in concussions and implications for service member health, cytokines levels of IL-6, IL-10, and TNF $\alpha$ were measured acutely in blood samples within $8 \mathrm{~h}$ following a medically diagnosed concussion and then again $24 \mathrm{~h}$ later to characterize the relationship between cytokines and recovery from acute brain injury. Findings from this line of research will provide the basis to identify the biological underpinnings of inflammatory processes occurring in the acute stage of recovery from concussion sustained in austere environments like military deployment, which is necessary to improve medical decision making and recovery trajectories.

\section{Methods}

\section{Participants}

This study protocol was reviewed and approved by the Research Institutional Review Boards at the US Army Medical Research and Development Command and the 
Walter Reed Army Institute of Research. Each study participant provided written informed consent for participation. This unique, observational cohort study consisted of: 1) deployed military personnel who sustained a concussion, provider diagnosed, without other major medical diagnosis and received acute medical care $(n=45)$ and 2) healthy control participants in the same deployment environment who did not sustain concussion or other illness or injuries $(n=49)$. Participants were referred to this protocol by treating providers as having concussion and no other major injury. Exclusion criteria for the control group included self-report of illness, pain, or history of head injury within previous 4 weeks. Both groups were deployed to units in the same region of operations in Afghanistan. The environment and process for diagnosis was comparable to a standard civilian emergency room. Concussion was defined as the reporting of an injury event, a normal structural neuroimaging by head CT or conventional brain MRI, and at least one of the following: alteration of consciousness less than $24 \mathrm{~h}$; loss of consciousness, if any, for less than $30 \mathrm{~min}$; or post-traumatic amnesia for less than $24 \mathrm{~h}$. Providers making diagnoses all held MD or DO degree and were working according to Department of Defense protocol [36].

Participants had blood draws at two time points: 1) time point 1 was at the time of medical care, less than $8 \mathrm{~h}$ after concussion, or at the time of initial encounter for the healthy control group and 2) time point 2 was $24 \mathrm{~h}$ following the time of the first blood draw. There was some attrition in each of the two cohorts between time point 1 and time point 2 . For this analysis, we included only participants with samples at both time points.

\section{Blood sampling}

Whole blood was drawn and processed for serum from April-November 2012 in Southwest Afghanistan, using standard protocols, within 1 hour of the blood draw. Serum was aliquoted and frozen on site at $80^{\circ} \mathrm{C}$. Frozen samples were shipped to the Walter Reed Army Institute of Research (WRAIR) in Maryland, USA at 4 separate time points across the collection period. Shipping was made with dry ice and a container designed for this purpose. Each shipment included a thermometer that continuously logged temperature during the shipment period. Upon receipt at WRAIR, shipments were transferred to continuously powered -80 freezers that are maintained according to WRAIR protocols. The final transfer of samples was approximately 5 miles from WRAIR to the National Institutes of Health, National Institute of Nursing Research (NINR) via insulated container and dry ice packing. Upon receipt at NINR, samples were transferred to NINR freezers and maintained according to protocol until thawed for assay from July to October 2017.

\section{Laboratory methods}

IL-6, IL-10, and TNF $\alpha$ concentrations were measured using Simoa ${ }^{\text {Tx }}$ technology (Quanterix, Lexington, MA), an ultrasensitive single-molecule enzyme-linked immunosorbent assay, as previously described [37]. The IL6, IL-10, and TNF $\alpha$ assays have low limits of detections $(0.006 \mathrm{pg} / \mathrm{mL}, 0.0022 \mathrm{pg} / \mathrm{mL}$, and $0.011 \mathrm{pg} / \mathrm{mL}$, respectively). Samples were run in duplicate, and the personnel running analyses were blinded to group. Average coefficients of variation $(\mathrm{CV})$ were $4.75,4.43$, and $4.78 \%$ for IL-6, IL-10 and TNF $\alpha$, respectively. Four samples with $\mathrm{CV}>15 \%$ were excluded from analysis, and are not included in the cohort sizes.

\section{Statistical methods}

SPSS version 25 (IBM Corporation, Chicago, IL) was used to conduct statistical analyses, and GraphPad Prism version 7.0d (Graph Pad Software, San Diego, CA) was used to create figures. Baseline demographic characteristics were compared between healthy and concussed groups using Pearson's chi square (race and gender) and ANOVA (age). Distributions did not require adjustment for normality. The differences in concentrations of IL-6, IL-10, and TNFa between the concussed and healthy groups at the first time point and at the second time point (time point $1=<8 \mathrm{~h}$ after injury; time point $2=24$ $\mathrm{h}$ following time point 1 ) were compared using Mann Whitney $U$ tests. For the difference between the two time points, mean difference was calculated by subtracting each participant's cytokine concentration at time point 2 from the concentration level at time point 1 , resulting in a variable that reflects the total change in each cytokine across $24 \mathrm{~h}$. A Mann Whitney U test was conducted to evaluate if there was a significant difference in time-based change for the cytokines between the concussed group and the healthy control group. Because the two groups were similar in demographic characteristics, we did not include race or age covariates in these models.

\section{Results}

\section{Demographics}

All participants were active duty service members $(n=$ 94) deployed to Afghanistan, and the sample was primarily male (96.8\%). The mean age was 26.41 years $(\mathrm{SD}=6.364)$ with a range of 19 to 48 years of age. Military personnel who were medically diagnosed with a concussion and received acute care $(n=45)$ were compared to healthy controls with no diagnosis of 
concussion $(n=49)$ deployed to the same combat station. The two groups did not differ in demographic features including sex, race, or age (see Table 1). All participants within both groups had a Glasgow Coma Score (GCS) of 15 at the time of study enrollment. The concussed personnel were evaluated and diagnosed by a healthcare provider according to standard of care and were then referred to study personnel $<8 \mathrm{~h}$ following injury. Of the concussed personnel, 33 (73.3\%) participants were exposed to blast during the injury event, and the others reported a blunt force injury without exposure to blast (see Table 2).

\section{Inflammatory protein differences following concussion Between-group comparisons at time point 1 and at time point 2}

Differences in IL-6, IL-10, and TNF $\alpha$ between concussed and healthy groups were evaluated at each time point (time point $1=<8 \mathrm{~h}$ after injury; time point $2=24 \mathrm{~h}$ following time point 1 ). At time point 1 , IL-6 median (interquartile range, IQR) concentrations were 2.62 (3.62) in the concussed group, which was greater compared to the IL-6 concentrations of $1.03(0.90)$ in the healthy control group $(U=420.00, \mathrm{z}=-5.12, p<0.001)$ (see Fig. 1a). The two highest levels of IL-6 were samples collected $>4 \mathrm{~h}$ after injury. Compared to healthy controls, the concussed group did not differ at time point 1 in concentrations of IL-10 $(p=0.358)$ or TNF $\alpha(p=$ 0.382 ) (see Fig. 1b-c). At time point 2, no differences were detected between concussed and healthy controls for IL-6 $(p=0.075)$, IL-10 $(p=0.937)$, or TNF $\alpha(p=$ 0.390) concentrations (see Fig. 1a-c). The median

Table 1 Demographic Data

\begin{tabular}{llll}
\hline & $\begin{array}{l}\text { Healthy Controls } \\
(N=49)\end{array}$ & $\begin{array}{l}\text { Concussion } \\
(N=45)\end{array}$ & Significance \\
\hline Age in years, M (SD) & $26.63(6.978)$ & $26.36(5.747)$ & $\begin{array}{l}p=0.841^{\mathrm{a}} \\
\mathrm{F}=0.041^{\mathrm{a}}\end{array}$ \\
$\begin{array}{l}\text { Sex, No. (\%) } \\
\text { Male }\end{array}$ & $49(100)$ & $42(93.3)$ & \\
Female & $0(0)$ & $3(6.7)$ & \\
Race, No. (\%) & & & \\
White & $35(71.4)$ & $21(67.7)$ & \\
Black & $5(10.2)$ & $0.297^{\mathrm{b}}$ \\
Hispanic & $6(12.2)$ & $5(7.5)$ & \\
Pacific Islander & $2(4.1)$ & $1(3.2)$ & \\
Asian & $1(2.0)$ & $1(3.2)$ & \\
Middle Eastern & $0(0.0)$ & $1(3.2)$ & \\
Other & $0(0.0)$ & $2(6.5)$ &
\end{tabular}

Note. The percentages in each column refer to the proportion of individuals in each sex and race category. Race for 14 participants in the concussed group is

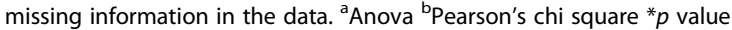
significant at the $p<0.05$ level
Table 2 Clinical Data

\begin{tabular}{ll}
\hline Reason for visit & Concussion $(N=45)$ \\
\hline Blast exposure, No. (\%) & $33(73.3)$ \\
Blunt force injury, without blast, No. (\%) & $12(26.7)$ \\
\hline
\end{tabular}

Note. The percentages in each column refer to the proportion of individuals with each reason for visit

difference between time point 1 and time point 2 was compared between the concussed and healthy control groups for IL-6, IL-10, and TNF $\alpha$. A Mann Whitney U test showed that the change in IL- 6 across time was greater for the concussed group than the healthy control $(-1.54$ (3.12); $\quad U=315.00, \quad \mathrm{z}=-5.96, \quad p<0.001) \quad$ (see Fig. 2a). There was no difference between groups in the change of IL-10 ( $p=0.158)$ or TNF $\alpha(p=0.777)$ (See Fig. $2 \mathrm{~b}-\mathrm{c})$. The percentage change in IL- 6 was $-67.7 \%$ in the concussed group compared to $33.5 \%$ in the healthy controls.

Within-group comparisons at time point 1 and time point 2 Within-group differences in IL-6, IL-10, and TNF $\alpha$ between concussed and healthy groups were evaluated at each time point using the Wilcoxon Sign-Rank Test. Change in IL- 6 between time points 1 and 2 in the concussed group remains statistically significant $(\mathrm{z}=-4.76$, $p<0.001$ ) (Fig. 3a). In the healthy control group, there was a significant increase in IL-6 from time point 1 to time point $2(\mathrm{z}=-3.17, p=0.002)$. However, the change was in the opposite direction for the concussed group and the effect size for the concussion group was larger (0.5) than for the healthy control group (0.3). There were no differences in the concussed group for IL-10 $(\mathrm{z}=-1.41, p=0.16)$ or TNF $\alpha(\mathrm{z}=-0.31, p=0.76)$, and there were no differences in the healthy control group for IL-10 $(\mathrm{z}=-0.58, p=0.56)$ or TNF $\alpha(\mathrm{z}=-1.46, p=$ 0.145 ) (Fig. 3b-c). 13.3\% (6 of the 45 individuals with concussion) of the concussed sample values at time point 1 were within the IQR of the healthy control group (0.68-1.58), and $80 \%$ (36 of the 45 individuals with concussion) of the concussed group was above the IQR of the healthy control group at time point 1 (>1.58).

\section{Area under the curve}

Binomial logistic regression provided individual IL-6, IL10 , TNF $\alpha$, and combined biomarker model ROC curves. To determine the ability of the cytokines to differentiate concussed and healthy control groupings at time point 1 , an area under the curve (AUC) analysis was performed (Fig. 4). IL-6 was a significant predictor and had a good AUC value (AUC 0.81, 95\% CI 0.72-0.90), and the combined biomarker model showed good discriminatory power (AUC 0.82, 95\% CI 0.73-0.90). IL-10 (AUC 0.56, 95\% CI 0.44-0.67) and TNF $($ AUC 0.55, 95\% CI 0.44-0.67) were not significant. 

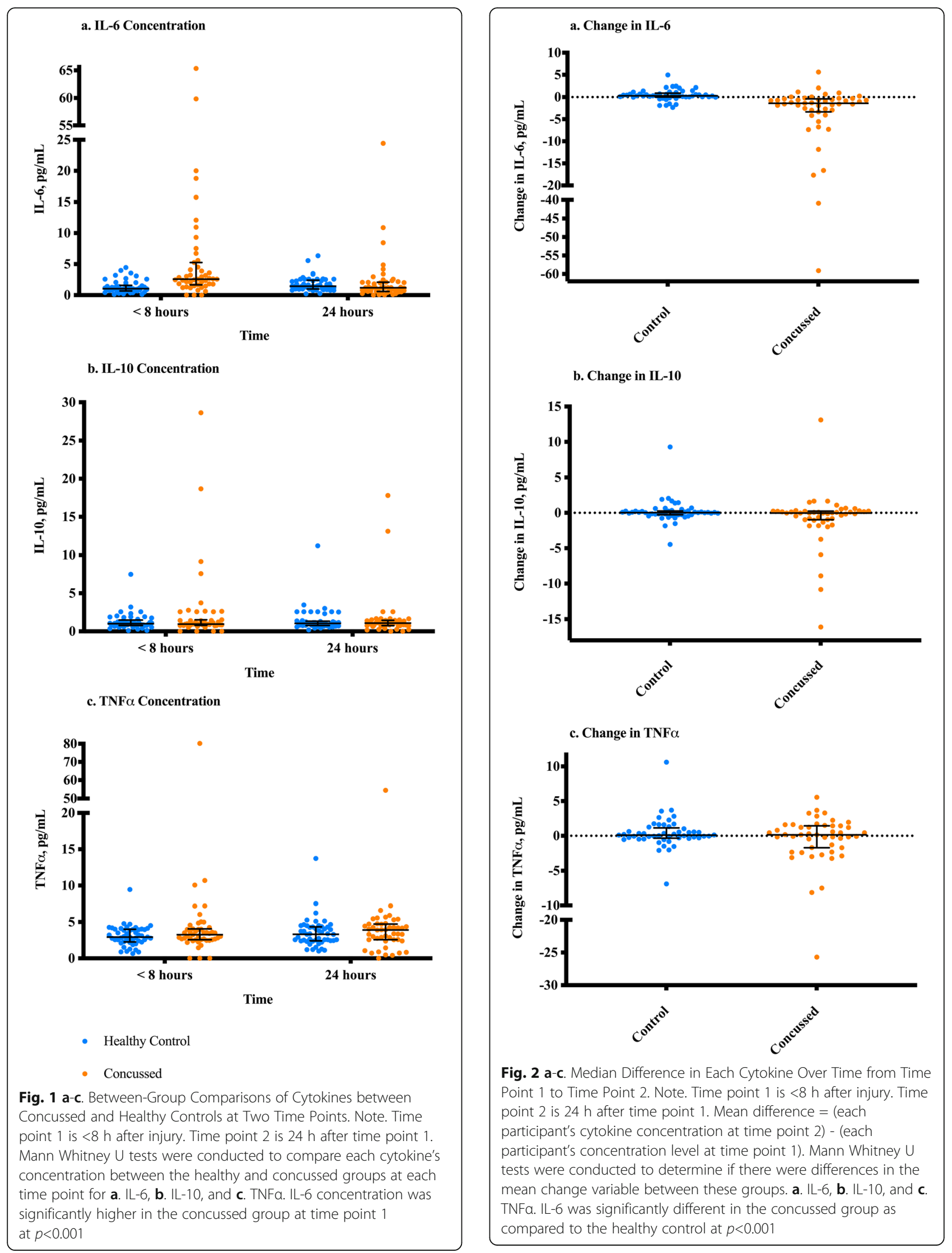
a. IL-6 Concentration

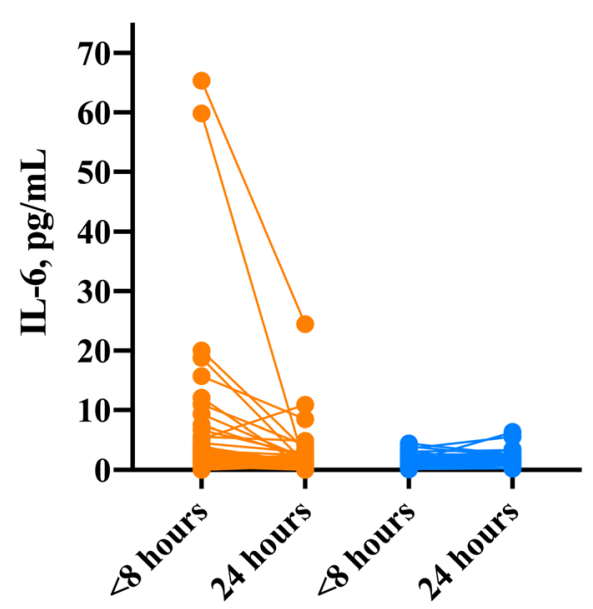

b. IL-10 Concentration

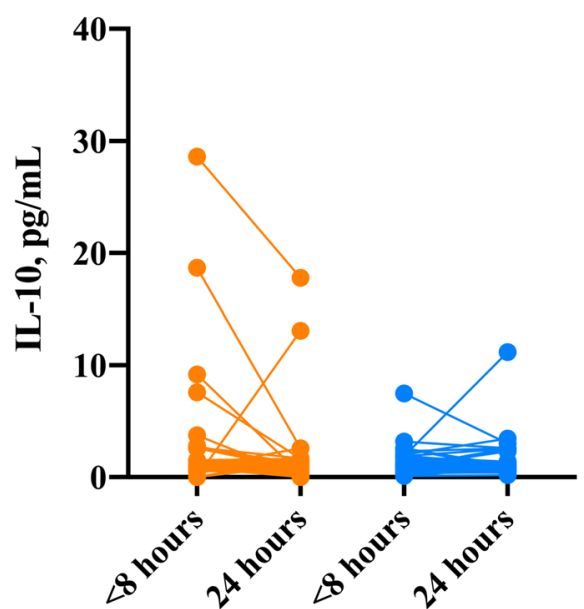

c. $\mathrm{TNF} \alpha$ Concentration

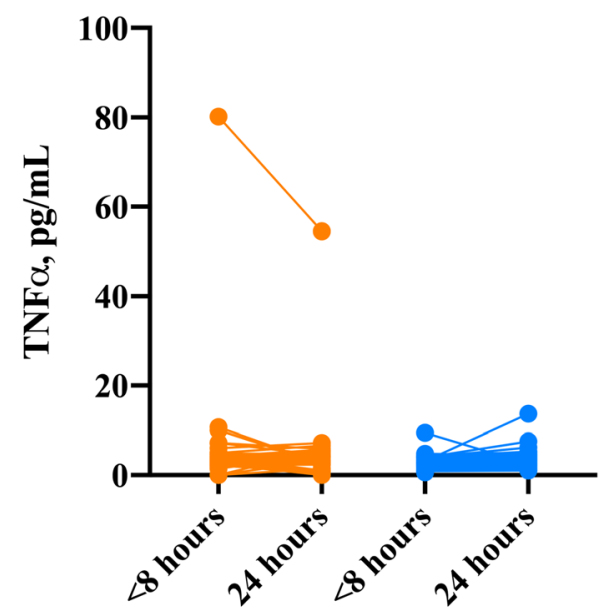

- Concussed

- Healthy Control

Fig. 3 a-c. Within-Group Comparisons of Cytokines between Concussed and Healthy Controls at Two Time Points. Note. Time point 1 is $<8 \mathrm{~h}$ after injury. Time point 2 is $24 \mathrm{~h}$ after time point 1. Within-groups differences were calculated used Wilcoxon Sign-Rank Tests comparing each cytokine's concentration between the healthy and concussed groups at each time point for $\mathbf{a}$. IL-6, b. IL-10, and $\mathbf{c}$. TNFa. IL-6 concentration was significantly higher in the concussed group at time point 1 as compared to time point $2(z=-4.76, p<0.001)$

\section{Discussion}

The finding of higher IL-6 within $8 \mathrm{~h}$ of a medically diagnosed concussion sustained during combat deployment is consistent with previous studies that report acutely elevated levels of IL-6 in severe TBI patients $[13,14,22]$. In fact, this study is the first one known that reports acute biomarker findings in a deployed cohort of military personnel with concussion. There are a variety of factors that make a deployed population unique, and for this study, paramount is the high rate of blast exposure. This finding is in line with this lab's previous report that linked IL-6 elevations to a blast exposure sustained during training by an undiagnosed population which did not include blunt force. That elevation was then followed by a decrease in IL-6 in sampling on subsequent days to below baseline levels [34]. Therefore, the present findings indicate that concussions sustained during deployment, highly comorbid with blast, result in elevations of IL-6, followed by a decrease in concentrations within $24 \mathrm{~h}$. This finding implicates a role for IL-6 in recovery from concussions, as well as blast exposures, and that understanding these complex relationships may be important to improving care provided to military personnel with complex, and often overlapping injuries sustained in combat stations.

IL-6 is involved in the modulation of inflammatory activity following a TBI, with evidence pointing to the 


\section{Concussed vs Control Groups}

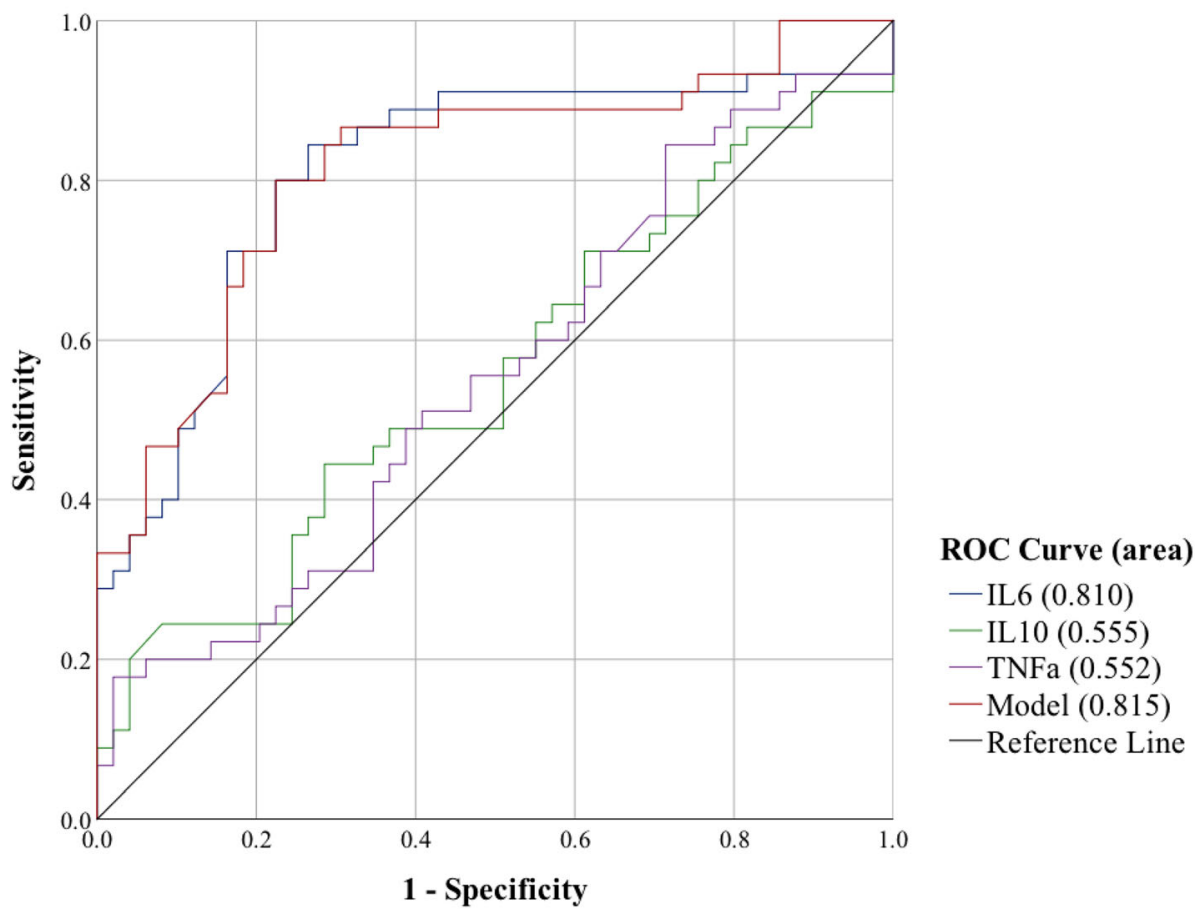

Fig. 4 Sensitivity of Acute Cytokines to Predict Concussed vs Control Groups. Note. Receiver operating characteristic (ROC) curves at time point 1 for IL-6, IL-10, TNFa, and the combined model which includes all biomarkers

importance of the balance of pro- and antiinflammatory levels in the promotion of recovery following TBIs and concussions [17]. Pro-inflammatory cytokines (including IL-1, IL-6, and TNF $\alpha$ ) and antiinflammatory cytokines (including IL-10 and IL-1 receptor agonist, IL-4) are released by a myriad of cells, including microglia, astrocytes, macrophages, and other peripheral and CNS cells, and orchestrate the acute inflammatory response to brain injury [11, 24, 38]. Anti-inflammatory cytokines shift the balance toward neuroregenerative and neuroprotective biological pathways, and pro-inflammatory cytokines shifting the balance toward apoptosis and cell death [38], and the threshold at which this balance becomes maladaptive is not fully understood [11]. Activation of microglia induces production of neuroprotective agents including IL-10 which suppresses the proinflammatory immune response [39]. In support of this inflammatory response balance, preclinical models that knock out IL-6 activity result in poor behavioral performance following a TBI [40] as well as increased apoptosis and delayed neuronal regeneration [20], implying an important pro-inflammatory function for IL-6 immediately following injury. However, detrimental outcomes do occur with microglial activation and elevated prolonged proinflammatory response [41]. IL-
6 activity following TBI has been linked to delays in motor coordination and neuronal tissue repair in preclinical models $[19,42]$. There is also evidence of increased IL-6 concentration in human post-mortem brain tissue obtained following a severe TBI that resulted in mortality, compared to patients who died from non-central nervous system causes [43]. There may be long-term health consequences that result if IL-6 remains imbalanced, as findings of elevated IL-6 may be indicative of chronic neurological symptoms or deficits [31]. Findings from the current study show an IL-6 elevation within hours of a concussion, with the highest two IL-6 levels more than $4 \mathrm{~h}$ after injury. Levels are then similar to healthy controls at $24 \mathrm{~h}$ later, suggesting that IL-6 is playing a role in recovery from these mild injuries. Considering these early findings in concussion, additional studies with longer follow up are warranted to understand the role of IL-6 in recovery and links to long-term consequences.

IL-10 and TNF $\alpha$ were not significantly different between the concussed and healthy cohorts in the present study. This differs from a previous report of elevated levels of TNF $\alpha$ in military personnel following blast exposure, along with elevated IL-6 levels [34]. One explanation may be differences between the samples in the two studies. The previous report studied military personnel 
in a well-controlled training environment, with no reported incidences of blunt injuries and no medical diagnosis [34], while the present cohort included military personnel diagnosed with a concussion, including from blunt force injury. These reported differences in event characteristics may account for a lack of TNF $\alpha$ differences between the concussed and healthy military personnel. Likewise, IL-10 was not significantly different between the groups in the present study, a finding that replicated these previous findings [34]. Elevations concurrently in IL- 6 and IL-10 have been observed in studies of severe TBI [13], and increases in serum IL-10 seem positively correlated with more severe TBI [23, 29]. The lack of an increase of IL-10 in the current concussed population is not surprising based on the previous literature. However, the lack of increase in IL-10 together with elevated IL- 6 observed in this cohort suggests a state of pro-inflammatory activation. IL-10 is traditionally classified as exerting anti-inflammatory effects, while IL-6 is traditionally defined as pro-inflammatory characteristics $[17,38,44]$, with some evidence for IL-6 anti-inflammatory characteristics [44]. IL-10 confers neuroprotective effects in animal models [4547]. Potential long-term outcomes if the proinflammatory response were to remain elevated should be further explored in larger sampling and with in-depth clinical measures.

Inflammatory markers, such as cytokines, may be valuable in modeling the recovery process and predicting outcomes following concussion. Inflammatory processes may modulate secondary pathological processes after TBI [11]. In contrast to the development of diagnostic biomarkers, which would have vital roles in point-of-care tests and screening $[10,48]$, a robust understanding of the inflammatory pathways, including the threshold for maladaptive response, may lead to insights into immunomodulated therapies and targets. The current study is a unique sample and the first study to report acute cytokine blood biomarkers obtained from active duty military personnel who sustained concussions during a military deployment.

There are a number of factors in the current study that limit interpretations of these findings, including a relatively small sample size. Baseline blood samples before head injury were not collected for this study, and further research efforts should include baseline levels to ensure it is the head injury exposure influencing changes in blood biomarkers. Inclusion of another control group, who sustained bodily injuries but not head injuries, would also be informative in future work to isolate the influence of head injury on blood cytokine levels. Additional limitations in the scope of this study include a lack of symptom data and evaluation of long-term outcomes. Since exclusion criteria relied upon self-report, previous exposures to low level blast exposure cannot be ruled out within the control or concussed cohorts. The nature of the combat environment may limit specificity in the current study, as blast exposure and blunt injuries often occur concurrently in the same injury event. Differences between injury types may account for discrepancies with the literature, though it is outside the scope of this study to delineate effects of blast from blunt force injury causes.

\section{Conclusion}

In conclusion, reported here is a significant elevation of IL-6 levels in concussed military personnel less than $8 \mathrm{~h}$ following injury. This is the first reported observation of blood levels IL-6, - 10, and TNF $\alpha$ in a combat environment to evaluate biomarker consequences of concussions sustained during combat deployments, which is a unique cohort given the high rates of comorbid blast exposure. The present finding of IL-6 elevation warrants further exploration of inflammatory cytokines in combat injuries involving concussion and blast, especially in future studies designed to account for the aforementioned limitations. Future studies may examine acute and chronic neurological symptomology associated with inflammatory cytokine levels, distinguish individuals at high risk for developing neurological complications, and identify underlying biological pathways to mitigate inflammation and improve outcomes. The present findings of elevated IL- 6 may be further explored in larger cohorts, as well as to determine inflammatory pathways that may be targeted for therapies.

\section{Abbreviations}

CT: Computerized tomography; CV: Coefficient of variation; IED: Improvised explosive device; IL-6: Interleukin-6; IL-10: Interleukin-10; OEF: Operation Enduring Freedom; TNFa: Tumor necrosis factor alpha; mTBI: Mild traumatic brain injury; TBI: Traumatic brain injury

\section{Acknowledgements}

The authors acknowledge the helpful comments provided by Dr. Kathleen Valentine, Dr. Julia Eggert, and Dr. Mary Beth Steck of the Clemson University Healthcare Genetics Program, and by Dr. Sheila Alexander of the University of Pittsburgh School of Nursing. The authors thank Dr. Jim McDonell of Clemson University for his statistical advice, and the U.S. military service members who volunteered to participate in this study.

\section{Authors' contributions}

$K A E, J M G$, WC contributed to the conception or design of the study; KAE, CL contributed to lab analysis and interpretation of the data; WC, MB, NR, DM, JE contributed to the collection, interpretation of the data and drafting and editing of the work; KAE, CP, and JMG contributed to the interpretation of the data and drafting and editing of the work. All authors contributed to critical revision of the manuscript, read and approved the submitted version, and agree to be accountable for all aspects of the work in ensuring that questions related to the accuracy or integrity of any part of the work are appropriately investigated and resolved. Accountable for the content of the work. 


\section{Funding}

This work was supported by the National Institute of Nursing Research (NINR) Intramural Program and the US Army Medical Research and Development Command (USAMRDC). NINR support was responsible for analysis, interpretation of data, and writing the manuscript. USAMRDC support was responsible for all aspects of this work, design of the study, collection, analysis, interpretation of data, and writing the manuscript. While producing this article, KAE was supported as a Jonas Nurse Leader Scholar. WC was supported in part by an appointment to the Research Participation Program at the Walter Reed Army Institute of Research administered by the Oak Ridge Institute for Science and Education through an interagency agreement between the U.S. Department of Energy and USAMRDC.

\section{Availability of data and materials}

The datasets analyzed during the current study available from the corresponding author on reasonable request.

\section{Ethics approval and consent to participate}

The study protocol was approved by the Walter Reed Army Institute of Research Institutional Review Boards and investigators adhered to the policies for protection of human subjects as prescribed in AR 70-25 and all applicable Federal regulations governing the protection of human subjects. Each study participant provided written informed consent for participation.

\section{Consent for publication}

Not applicable.

\section{Competing interests}

The authors declare that the research was conducted in the absence of any commercial or financial relationships that could be construed as a potential conflict of interest. The opinions and assertions in this manuscript are those of the authors and are not to be construed as official as reflecting true views of the Department of Navy, Department of the Air Force, Department of the Army, Department of Defense, the Uniformed Services University of the Health Sciences, U.S. Government, the Center for Neuroscience and Regenerative Medicine or any other agency of the U.S. government. Some of the authors are military service members or employees of the U.S. Government. This work was prepared as part of their official duties. Title 17 U.S.C. \$105 provides that 'Copyright protection under this title is not available for any work of the United States Government.' Title 17 U.S.C. \$101 defines a U.S. Government work as a work prepared by a military service member or employee of the U.S. Government as part of that person's official duties. Material has been reviewed by the Walter Reed Army Institute of Research. There is no objection to its presentation and/or publication.

\section{Author details}

${ }^{1}$ National Institute of Nursing Research, National Institutes of Health, 3 Center Drive, Building 3, Room 26E, Bethesda, MD 20892, USA. ${ }^{2}$ Henry M. Jackson Foundation for the Advancement of Military Medicine, 6720A Rockledge Dr, Bethesda, MD 20817, USA. ${ }^{3}$ CNRM Co-Director Biomarkers Core, Uniformed Services University of the Health Sciences, Bethesda, USA. ${ }^{4} 87$ th Medical Group, Joint Base McGuire-Dix-Lakehurst, 3458 Neely Road, Trenton, NJ 08641, USA. ${ }^{5}$ United States Army Research Institute of Environmental Medicine, 10 General Greene Ave, Natick, MA 01760, USA. ' USS Gerald R. Ford (CVN78), FPO, AE, Norfolk, VA 09523, USA. ${ }^{7}$ Oak Ridge Institute for Science and Education, Oak Ridge, TN, USA. ${ }^{8}$ Walter Reed Army Institute of Research, 503 Robert Grant Avenue, Silver Spring, MD 20910, USA.

\section{Received: 16 August 2019 Accepted: 3 May 2020}

Published online: 25 May 2020

\section{References}

1. Jones E, Fear NT, Wessely S. Shell shock and mild traumatic brain injury: a historical review. Am J Psychiatry. 2007;164(11):1641-5.

2. DePalma RG. Frontiers in Neuroengineering.Combat TBI: History, Epidemiology, and Injury Modes. In: Kobeissy FH, editor. Brain Neurotrauma: Molecular, Neuropsychological, and Rehabilitation Aspects. Boca Raton: CRC Press/Taylor \& Francis.(c) 2015 by Taylor \& Francis Group, LLC; 2015.
3. Prieto DA, Ye $X$, Veenstra TD. Proteomic analysis of traumatic brain injury: the search for biomarkers. Expert Rev Proteomics. 2008;5(2):28391.

4. Meerlo P, Sgoifo A, Suchecki D. Restricted and disrupted sleep: effects on autonomic function, neuroendocrine stress systems and stress responsivity. Sleep Med Rev. 2008;12(3):197-210.

5. Ritenour AE, Baskin TW. Primary blast injury: update on diagnosis and treatment. Crit Care Med. 2008;36(7 Suppl):S311-7.

6. Mac Donald CL, Johnson AM, Cooper D, Nelson EC, Werner NJ, Shimony JS, et al. Detection of blast-related traumatic brain injury in U.S. military personnel. N Engl J Med. 2011;364(22):2091-100.

7. Mac Donald CL, Johnson AM, Wierzechowski L, Kassner E, Stewart T, Nelson EC, et al. Outcome trends after US military concussive traumatic brain injury. J Neurotrauma. 2017;34(14):2206-19.

8. Mac Donald CL, Johnson AM, Wierzechowski L, Kassner E, Stewart T, Nelson EC, et al. Prospectively assessed clinical outcomes in concussive blast vs nonblast traumatic brain injury among evacuated US military personnel. JAMA neurology. 2014;71(8):994-1002.

9. Carr W, Polejaeva E, Grome A, Crandall B, LaValle C, Eonta SE, et al. Relation of repeated low-level blast exposure with symptomology similar to concussion. J Head Trauma Rehabil. 2015;30(1):47-55.

10. Bazarian JJ, Biberthaler $P$, Welch RD, Lewis LM, Barzo P, Bogner-Flatz V, et al. Serum GFAP and UCH-L1 for prediction of absence of intracranial injuries on head CT (ALERT-TBI): a multicentre observational study. Lancet Neurol. 2018;17(9):782-9.

11. Hinson HE, Rowell S, Schreiber M. Clinical evidence of inflammation driving secondary brain injury: a systematic review. J Trauma Acute Care Surg. 2015 78(1):184-91.

12. Ng SY, Lee AYW. Traumatic brain injuries: pathophysiology and potential therapeutic targets. Front Cell Neurosci. 2019;13:528.

13. Ferreira LC, Regner A, Miotto KD, Moura S, Ikuta N, Vargas $A E$, et al. Increased levels of interleukin-6, -8 and -10 are associated with fatal outcome following severe traumatic brain injury. Brain Inj. 2014;28(10): 1311-6.

14. Woiciechowsky C, Schoning B, Cobanov J, Lanksch WR, Volk HD, Docke WD. Early IL-6 plasma concentrations correlate with severity of brain injury and pneumonia in brain-injured patients. J Trauma. 2002;52(2): $339-45$.

15. Kumar RG, Boles JA, Wagner AK. Chronic inflammation after severe traumatic brain injury: characterization and associations with outcome at 6 and 12 months Postinjury. J Head Trauma Rehabil. 2015;30(6):369-81.

16. Licastro F, Hrelia S, Porcellini E, Malaguti M, Di Stefano C, Angeloni C, et al. Peripheral inflammatory markers and antioxidant response during the postacute and chronic phase after severe traumatic brain injury. Front Neurol. 2016:7:189.

17. McKee CA, Lukens JR. Emerging roles for the immune system in traumatic brain injury. Front Immunol. 2016;7:556.

18. Woodcock T, Morganti-Kossmann MC. The role of markers of inflammation in traumatic brain injury. Front Neurol. 2013;4:18.

19. Penkowa M, Giralt M, Lago N, Camats J, Carrasco J, Hernandez J, et al. Astrocyte-targeted expression of IL-6 protects the CNS against a focal brain injury. Exp Neurol. 2003;181(2):130-48.

20. Penkowa M, Giralt M, Carrasco J, Hadberg H, Hidalgo J. Impaired inflammatory response and increased oxidative stress and neurodegeneration after brain injury in interleukin-6-deficient mice. Glia. 2000;32(3):271-85

21. Kline $A E$, Bolinger BD, Kochanek PM, Carlos TM, Yan HQ, Jenkins LW, et al. Acute systemic administration of interleukin-10 suppresses the beneficial effects of moderate hypothermia following traumatic brain injury in rats. Brain Res. 2002;937(1-2):22-31.

22. Arand M, Melzner H, Kinzl L, Bruckner UB, Gebhard F. Early inflammatory mediator response following isolated traumatic brain injury and other major trauma in humans. Langenbeck's Arch Surg. 2001;386(4):241-8.

23. Di Battista AP, Rhind SG, Hutchison MG, Hassan S, Shiu MY, Inaba K, et al. Inflammatory cytokine and chemokine profiles are associated with patient outcome and the hyperadrenergic state following acute brain injury. J Neuroinflammation. 2016;13:40.

24. Helmy A, Carpenter KL, Menon DK, Pickard JD, Hutchinson PJ. The cytokine response to human traumatic brain injury: temporal profiles and evidence for cerebral parenchymal production. J Cereb Blood Flow Metab. 2011;31(2): 658-70. 
25. Hergenroeder GW, Moore AN, McCoy JP Jr, Samsel L, Ward NH 3rd, Clifton $\mathrm{GL}$, et al. Serum IL-6: a candidate biomarker for intracranial pressure elevation following isolated traumatic brain injury. J Neuroinflammation. 2010;7:19.

26. Juengst SB, Kumar RG, Failla MD, Goyal A, Wagner AK. Acute inflammatory biomarker profiles predict depression risk following moderate to severe traumatic brain injury. J Head Trauma Rehabil. 2015;30(3):207-18.

27. Lustenberger T, Kern M, Relja B, Wutzler S, Stormann P, Marzi I. The effect of brain injury on the inflammatory response following severe trauma. Immunobiology. 2016;221(3):427-31.

28. Yousefzadeh-Chabok S, Dehnadi Moghaddam A, Kazemnejad-Leili E, Saneei Z, Hosseinpour M, Kouchakinejad-Eramsadati L, et al. The relationship between serum levels of interleukins 6, 8, 10 and clinical outcome in patients with severe traumatic brain injury. Arch Trauma Res. 2015;4(1): e18357.

29. Schneider Soares FM, Menezes de Souza N, Liborio Schwarzbold M, Paim Diaz A, Costa Nunes J, Hohl A, et al. Interleukin-10 is an independent biomarker of severe traumatic brain injury prognosis. Neuroimmunomodulation. 2012;19(6):377-85.

30. Nitta ME, Savitz J, Nelson LD, Teague TK, Hoelzle JB, McCrea MA, et al. Acute elevation of serum inflammatory markers predicts symptom recovery after concussion. Neurology. 2019;93(5):e497-507.

31. Devoto C, Arcurio L, Fetta J, Ley M, Rodney T, Kanefsky RZ, et al. Inflammation relates to chronic behavioral and neurological symptoms in military with traumatic brain injuries. Cell Transplant. 2016;26(7):1169-77.

32. Di Battista AP, Churchill N, Rhind SG, Richards D, Hutchison MG. Evidence of a distinct peripheral inflammatory profile in sport-related concussion. J Neuroinflammation. 2019;16(1):17.

33. Sun Y, Bai L, Niu X, Wang Z, Yin B, Bai G, et al. Elevated serum levels of inflammation-related cytokines in mild traumatic brain injury are associated with cognitive performance. Front Neurol. 2019;10:1120.

34. Gill J, Motamedi V, Osier N, Dell K, Arcurio L, Carr W, et al. Moderate blast exposure results in increased IL-6 and TNFalpha in peripheral blood. Brain Behav Immun. 2017:65:90-4.

35. Menon DK, Schwab K, Wright DW, Maas Al. Position statement: definition of traumatic brain injury. Arch Phys Med Rehabil. 2010;91(11):1637-40.

36. Conaton E. DoD policy guidance for management of mild traumatic brain injury/concussion in the deployed setting. Washington: Department of Defense; 2012

37. Mondello S, Buki A, Barzo P, Randall J, Provuncher G, Hanlon D, et al. CSF and plasma amyloid-beta temporal profiles and relationships with neurological status and mortality after severe traumatic brain injury. Sci Rep. 2014;4:6446.

38. Hernandez-Ontiveros DG, Tajiri N, Acosta S, Giunta B, Tan J, Borlongan CV. Microglia activation as a biomarker for traumatic brain injury. Front Neurol. 2013;4:30.

39. Garcia JM, Stillings SA, Leclerc JL, Phillips H, Edwards NJ, Robicsek SA, et al. Role of Interleukin-10 in acute brain injuries. Front Neurol. 2017;8:244.

40. Ley EJ, Clond MA, Singer MB, Shouhed D, Salim A. IL6 deficiency affects function after traumatic brain injury. J Surg Res. 2011;170(2):253-6.

41. Ramlackhansingh AF, Brooks DJ, Greenwood RJ, Bose SK, Turkheimer FE, Kinnunen KM, et al. Inflammation after trauma: microglial activation and traumatic brain injury. Ann Neurol. 2011;70(3):374-83.

42. Yang SH, Gangidine M, Pritts TA, Goodman MD, Lentsch AB. Interleukin 6 mediates neuroinflammation and motor coordination deficits after mild traumatic brain injury and brief hypoxia in mice. Shock (Augusta, Ga). 2013; 40(6):471-5

43. Frugier T, Morganti-Kossmann MC, O'Reilly D, McLean CA. In situ detection of inflammatory mediators in post mortem human brain tissue after traumatic injury. J Neurotrauma. 2010;27(3):497-507.

44. Brandt C, Pedersen BK. The role of exercise-induced myokines in muscle homeostasis and the defense against chronic diseases. J Biomed Biotechnol. 2010;2010:520258.

45. Chen X, Duan XS, Xu LJ, Zhao JJ, She ZF, Chen WW, et al. Interleukin-10 mediates the neuroprotection of hyperbaric oxygen therapy against traumatic brain injury in mice. Neuroscience. 2014;266:235-43.

46. Zou R, Li D, Wang G, Zhang M, Zhao Y, Yang Z. TAZ activator is involved in IL-10-mediated muscle responses in an animal model of traumatic brain injury. Inflammation. 2017:40(1):100-5.

47. Barrett JP, Henry RJ, Villapol S, Stoica BA, Kumar A, Burns MP, et al. NOX2 deficiency alters macrophage phenotype through an IL-10/STAT3 dependent mechanism: implications for traumatic brain injury. J Neuroinflammation. 2017;14(1):65.

48. Papa L. Potential blood-based biomarkers for concussion. Sports Med Arthrosc Rev. 2016;24(3):108-15.

\section{Publisher's Note}

Springer Nature remains neutral with regard to jurisdictional claims in published maps and institutional affiliations.
Ready to submit your research? Choose BMC and benefit from:

- fast, convenient online submission

- thorough peer review by experienced researchers in your field

- rapid publication on acceptance

- support for research data, including large and complex data types

- gold Open Access which fosters wider collaboration and increased citations

- maximum visibility for your research: over $100 \mathrm{M}$ website views per year

At $\mathrm{BMC}$, research is always in progress.

Learn more biomedcentral.com/submissions 